\title{
Dickkopf-1/cysteine-rich angiogenic inducer 61 axis mediates palmitic acid-induced inflammation and apoptosis of vascular endothelial cells
}

\author{
YI-RONG GAN $^{1 *}$, LING WEI $^{2 *}$, YAN-ZHEN WANG $^{1}$, ZONG-KE KOU $^{1}$, TIAN-XIANG LIANG ${ }^{1}$, \\ GUAN-WANER DING ${ }^{3}$, YAN-HONG DING ${ }^{4}$ and DING-XIONG XIE ${ }^{1,5}$ \\ ${ }^{1}$ Gansu Cardiovascular Institute; ${ }^{2}$ Department of Outpatient, The First People's Hospital of Lanzhou City, \\ Lanzhou, Gansu 730050; ${ }^{3}$ Department of Clinical Medicine, Shijiazhuang People's Medical College, Shijiazhuang, \\ Hebei 050599; ${ }^{4}$ Department of Anesthesiology, The First People's Hospital of Lanzhou City, Lanzhou, Gansu 730050; \\ ${ }^{5}$ Department of Cardiac Surgery, Lanzhou University Second Hospital, Lanzhou, Gansu 730030, P.R. China
}

Received June 8, 2020; Accepted October 19, 2020

DOI: $10.3892 / \mathrm{mmr} .2020 .11761$

\begin{abstract}
Cardiovascular diseases (CVDs) are a major cause of mortality around the world, and the presence of atherosclerosis is the most common characteristic in patients with CVDs. Cysteine-rich angiogenic inducer 61 (CCN1) has been reported to serve an important role in the pathogenesis of atherosclerotic lesions. The aim of the present study was to investigate whether $\mathrm{CCN} 1$ could regulate the inflammation and apoptosis of endothelial cells induced by palmitic acid (PA). Dickkopf-1 (DKK1) is an important antagonist of the Wnt signaling pathway, which can specifically inhibit the classic Wnt signaling pathway. Firstly, the mRNA and protein expression levels of CCN1 were detected. Additionally, endothelial nitric oxide (NO) synthase (eNOS), DKK1, $\beta$-catenin, and inflammation- and apoptosis-associated proteins were measured. Detection of NO was performed using a commercial kit. The expression levels of inflammatory cytokines were assessed to explore the effect of $\mathrm{CCN} 1$ on PA-induced inflammation. TUNEL assay was used to detect the apoptosis of endothelial cells. The results revealed that PA upregulated the expression levels of CCN1, inflammatory cytokines and pro-apoptotic proteins in endothelial cells. PA decreased the production of NO, and the levels of phosphorylated-eNOS,
\end{abstract}

Correspondence to: Dr Yan-Hong Ding, Department of Anesthesiology, The First People's Hospital of Lanzhou City, 1 Wujiayuan West Street, Qilihe, Lanzhou, Gansu 730050, P.R. China E-mail: dyanhong05@163.com

Dr Ding-Xiong Xie, Gansu Cardiovascular Institute, 1 Wujiayuan West Street, Qilihe, Lanzhou, Gansu 730050, P.R. China

E-mail: xiedingxiong45@126.com

*Contributed equally

Key words: Dickkopf-1, cardiovascular diseases, cysteine-rich angiogenic inducer 61, human umbilical vein endothelial cells whereas knockdown of CCN1 partially abrogated these effects triggered by PA. Furthermore, the Wnt/ $\beta$-catenin signaling pathway was activated in PA-induced endothelial cells; however, the levels of DKK1 were downregulated. Overexpression of DKK1 could reduce CCN1 expression via inactivation of the Wnt/ $\beta$-catenin signaling pathway. In conclusion, knockdown of CCN1 attenuated PA-induced inflammation and apoptosis of endothelial cells via inactivating the $\mathrm{Wnt} / \beta$-catenin signaling pathway.

\section{Introduction}

Cardiovascular diseases (CVDs) are a major cause of mortality worldwide, and atherosclerosis is a chronic CVD characterized by the hardening and narrowing of arteries, within which are plaques that contain inflammatory cells, lipids, dead endothelial cells and proliferated vascular smooth muscle cells $(1,2)$. Atherosclerosis is the primary cause of mortality in CVDs due to its clinical manifestations, including stroke and coronary heart disease (3). Despite advances in the knowledge of atherosclerosis over recent years, the multiple risk factors and the complex mechanisms for this disease have resulted in difficulties in the diagnosis and treatment of atherosclerosis. Thus, understanding the mechanisms underlying atherosclerosis is required to optimize clinical interventions (4).

Cysteine-rich angiogenic inducer 61 (CCN1) belongs to the $\mathrm{CCN}$ family, which is a group of matricellular proteins secreted by endothelial cells and fibroblasts (5). CCN1 has been demonstrated to serve a role in leukocyte migration, inflammation and cardiovascular development $(5,6)$. CCN1 was revealed to be predominantly expressed in the atherosclerotic aortas of apolipoprotein $\mathrm{E}^{-/-}$mice, and $\mathrm{CCN} 1$ treatment deteriorated hyperlipidemia, systemic inflammation and the progression of atherosclerosis (7). In macrophages, neutralizing antibody or small interfering RNA (siRNA) could inhibit the activity of CCN1, thereby attenuating oxidized low-density lipoprotein (ox-LDL)-induced lipid accumulation (7). Furthermore, CCN1 has been shown to promote apoptosis of endothelial cells in the presence of TNF- $\alpha$ (2). 
Fatty acids (FAs) can be classified into three major types: Short-, medium- and long-chain FAs (SCFAs, MCFAs and LCFAs, respectively). A number of studies have demonstrated that, in contrast with SCFAs and MCFAs, LCFAs bear greater risks for the occurrence of coronary heart disease, which is one of the major types of CVD $(8,9)$. Palmitic acid (PA), which falls under the category of LCFAs, is the most common saturated FA in food, plants and animal products. PA has been reported to be involved in the apoptotic process of various cells, including cardiomyocytes and endothelial cells (10-13). Furthermore, a previous plasma metabolomic study has identified PA as a novel biomarker of atherosclerosis (14). However, little is currently known about the role of CCN1 in PA-induced endothelial cell injury. Human umbilical vein endothelial cells (HUVECs) are widely used to study the functions of endothelial cells (15-17). The present study aimed to explore the mechanism by which $\mathrm{CCN} 1$ exerts its effects on the inflammation and apoptosis of PA-induced HUVECs.

\section{Materials and methods}

Cell culture. The HUVEC line used in the present study was obtained from Shanghai Cell Resource Center, Shanghai Institutes for Biological Sciences, Chinese Academy of Sciences. The cells were cultured in Dulbecco's modified Eagle's medium (Gibco; Thermo Fisher Scientific, Inc.) supplemented with $10 \%$ fetal bovine serum (Gibco; Thermo Fisher Scientific, Inc.) at $37^{\circ} \mathrm{C}$ in an atmosphere containing 5\% $\mathrm{CO}_{2}$. PA (Sigma-Aldrich; Merck $\mathrm{KGaA}$ ) was dissolved in $0.1 \mathrm{mM}$ sodium hydroxide at $70^{\circ} \mathrm{C}$ and combined with $10 \%$ fatty acid-free BSA (Beijing Solarbio Science \& Technology Co., Ltd.) at $55^{\circ} \mathrm{C}$ for $10 \mathrm{~min}$ to achieve the final concentrations. The obtained PA $(0.2,0.4$ and $0.8 \mathrm{mM})$ was used to stimulate HUVECs for $24 \mathrm{~h}$ at $37^{\circ} \mathrm{C}$.

Cell transfection. siRNAs targeting CCN1 and Dickkopf-1 (DKK1) (CCN1 siRNA\#1 and CCN1 siRNA\#2; DKK1 siRNA\#1 and DKK1 siRNA\#2, respectively) and a negative control siRNA (control siRNA) were synthesized by Guangzhou RiboBio Co., Ltd. DKK1 overexpression plasmids (OE-DKK1) and negative control plasmids (empty pCEP4 vector; OE-NC) were provided by Shanghai GenePharma Co., Ltd. HUVECs (1x10 6 cells/well) were incubated at $37^{\circ} \mathrm{C}$ until they reached $70-80 \%$ confluence, and were transfected with $30 \mathrm{nM}$ siRNA or $20 \mu \mathrm{g}$ plasmids using Lipofectamine ${ }^{\circledR} 2000$ (Invitrogen; Thermo Fisher Scientific, Inc.) according to the manufacturer's instructions. A total of $48 \mathrm{~h}$ post-transfection, cells were collected to verify transfection efficiency. Transfected cells were then treated with $0.8 \mathrm{mM}$ PA for $24 \mathrm{~h}$ at $37^{\circ} \mathrm{C}$ in subsequent experiments. The sequences are shown in Table SI.

Reverse transcription-quantitative PCR (RT-qPCR). Total RNA was isolated from transfected HUVECs using TRIzol $^{\circledR}$ (Invitrogen; Thermo Fisher Scientific, Inc.). The PrimeScript RT Master Mix kit (Takara Biotechnology, Co., Ltd.) was utilized to synthesize cDNA according to the manufacturer's instructions. Subsequently, qPCR was performed with SYBR-Green PCR Master mix (Roche Diagnostics) on an ABI quantitative PCR 7500 system (Applied Biosystems; Thermo Fisher Scientific, Inc.). Thermocycling conditions were as follows: $95^{\circ} \mathrm{C}$ for $10 \mathrm{~min}$, followed by 40 cycles at $95^{\circ} \mathrm{C}$ for $15 \mathrm{sec}$ and $60^{\circ} \mathrm{C}$ for $60 \mathrm{sec}$. At the end of the reaction, the melting curve was analyzed. Relative gene expression was normalized to $\beta$-actin, and the associated data were normalized using the $2^{-\triangle \Delta C q}$ method (18). The primer sequences are listed in Table SII.

Western blotting. Total proteins were extracted from cells using RIPA lysis buffer (Beyotime Institute of Biotechnology) containing proteasome inhibitors. All protein samples were quantified using a BCA protein assay kit (Beyotime Institute of Biotechnology) and $20 \mu \mathrm{g}$ protein was subjected to SDS-PAGE on $10 \%$ gels. Proteins were then transferred onto polyvinylidene fluoride membranes (Bio-Rad Laboratories, Inc.). Subsequently, the membranes were blocked with $5 \%$ non-fat milk in Tris-buffered saline containing $0.1 \%$ Tween-20 at room temperature for $1 \mathrm{~h}$, and were then incubated with primary antibodies at $4^{\circ} \mathrm{C}$ overnight. The following primary antibodies were used: Anti-CCN1 (1:3,000; cat. no. ab228592), anti-IKK- $\beta$ (1:5,000; cat. no. ab124957), anti-NF- $\mathrm{B}(1: 5,000$; cat. no. ab32536), anti-phosphorylated (p)-IKK- $\beta$ (1:1,000; cat. no. ab59195), anti-p-NF-кB (1:1,000; cat. no. ab194726), anti-endothelial nitric oxide (NO) synthase (eNOS; 1:1,000; cat. no. ab76198), anti-p-eNOS (1:2,000; cat. no. ab184154), anti-caspase-3 (1:1,000; cat. no. ab90437), anti-cleaved caspase-3 (1:500; cat. no. ab2302) (all from Abcam), anti-Bcl-2 (1:1,000; cat. no. 15071), anti-Bax (1:1,000; cat. no. 2772), anti- $\beta$-catenin (1:1,000; cat. no. 9562), anti-DKK1 (1:1,000; cat. no. 4687) and anti- $\beta$-actin $(1: 1,000$; cat. no. 8457) (all from Cell Signaling Technology, Inc.). Subsequently, the membranes were incubated with horseradish peroxidase-conjugated anti-mouse and anti-rabbit IgG secondary antibodies (1:1,000; cat. nos. A0216 and A0208; Beyotime Institute of Biotechnology) at room temperature for $1 \mathrm{~h}$ and visualized using an electrochemiluminescence system (Amersham; Cytiva). Protein expression levels were semi-quantified using Image-Pro Plus software version 6.0 (Media Cybernetics, Inc.).

Measurement of NO production. The detection of NO was performed using NO detection kits (cat. no. A012-1-2; Nanjing Jiancheng Bioengineering Institute) according to the manufacturer's protocol.

ELISA. Cell medium of each group was subjected to centrifugation $(500 \mathrm{x} \mathrm{g}, 5 \mathrm{~min})$ at room temperature. The levels of inflammatory cytokines, including TNF- $\alpha$, IL-1 $\beta$ and IL-6, in culture media were detected using the TNF- $\alpha$ ELISA kit (cat. no. F0281), L-1 $\beta$ ELISA kit (cat. no. F0122) and IL-6 ELISA kit (cat. no. F01310) according to the manufacturer's protocols (Shanghai Xi Tang Biological Technology Co., Ltd.). Absorbance at $450 \mathrm{~nm}$ was detected using a microplate reader (Bio-Tek Corporation).

TUNEL assay. Cell apoptosis was detected using a TUNEL Assay kit (Beyotime Institute of Biotechnology) according the manufacturer's protocol. Briefly, cells in a 24-well plate ( $2 \times 10^{5}$ cells/well) were fixed with $4 \%$ paraformaldehyde for $30 \mathrm{~min}$ at room temperature, treated with PBS containing $0.3 \%$ Triton $\mathrm{X}-100$ and incubated at room temperature for 
A

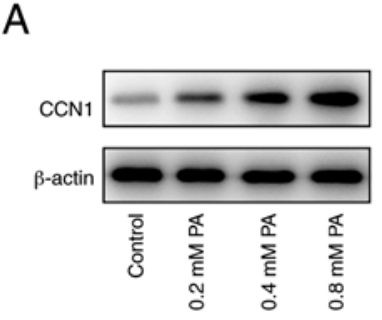

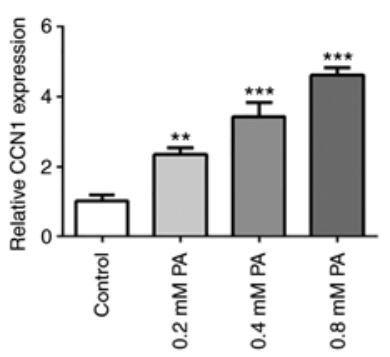
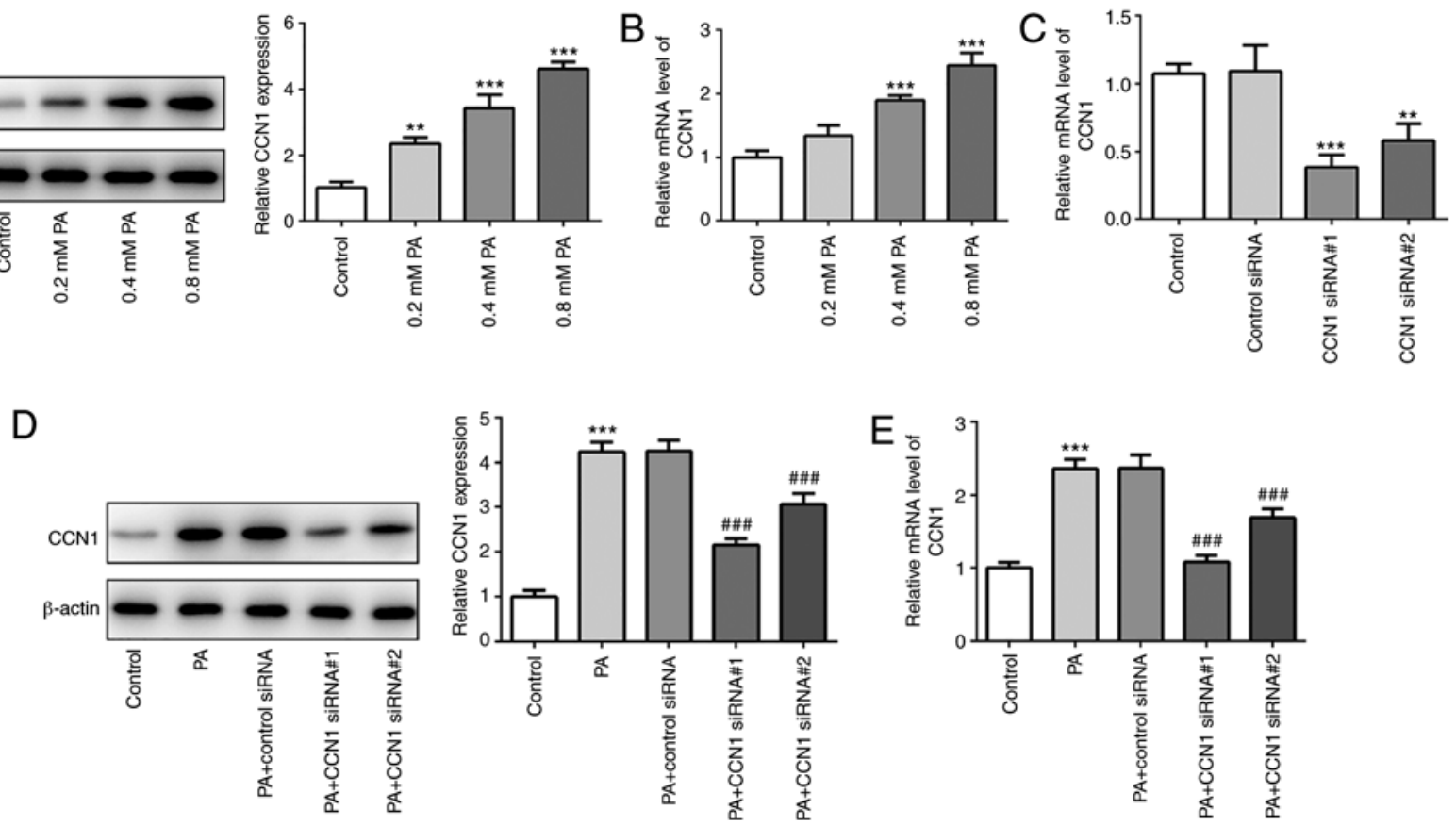

Figure 1. (A) Protein and (B) mRNA expression levels of CCN1 in HUVECs exposed to $0.2,0.4$ and $0.8 \mathrm{mM}$ PA for $24 \mathrm{~h} .{ }^{* * *} \mathrm{P}<0.01,{ }^{* * * *} \mathrm{P}<0.001$ vs. control group (C) mRNA expression levels of CCN1 in HUVECs transfected with CCN1 siRNA. ${ }^{* *} \mathrm{P}<0.01,{ }^{* * *} \mathrm{P}<0.001$ vs. control siRNA group. (D) Protein and (E) mRNA expression levels of CCN1 in HUVECs exposed to $0.8 \mathrm{mM}$ PA with or without siRNA. ${ }^{* * * *} \mathrm{P}<0.001$ vs. control group; ${ }^{\# \# \#} \mathrm{P}<0.001 \mathrm{vs}$. PA + control siRNA group. PA, palmitic acid; CCN1, cysteine-rich angiogenic inducer 61; HUVECs, human umbilical vein endothelial cells; siRNA, small interfering RNA.

5 min. Following the addition of $50 \mu 1$ TUNEL detection solution to the sample and incubation at $37^{\circ} \mathrm{C}$ for $60 \mathrm{~min}$ in the dark, cells were washed with PBS. Finally, the apoptotic cells were observed under a fluorescence microscope (magnification, x100; Olympus Corporation) after mounting with an anti-fluorescence quenching mounting solution.

Statistical analysis. Data are presented as the mean \pm standard deviation. SPSS 17.0 statistical software (SPSS, Inc.) was used for all statistical analyses. Each experiment was performed in triplicate. Comparisons between groups were analyzed by one-way ANOVA followed by Tukey's test. $\mathrm{P}<0.05$ was considered to indicate a statistically significant difference.

\section{Results}

Expression of CCN1 in PA-induced HUVECs. To confirm the effects of PA on CCN1 expression, the expression levels of CCN1 were measured in in PA-induced HUVECs. As presented in Fig. 1A and B, the mRNA and protein expression levels of CCN1 were gradually elevated in HUVECs treated with increasing concentrations of PA compared with those in the control group. The results revealed that the expression of CCN1 was elevated in PA-induced HUVECs in a dose-dependent manner. PA at a concentration of $0.8 \mathrm{mM}$ was used for further experiments. Subsequently, CCN1 was silenced via transfection with a siRNA (Fig. 1C). As presented in Fig. 1D and E, the expression levels of CCN1 were significantly elevated in PA-induced HUVECs compared with those in the control group, whereas this effect was reversed when CCN1 was knocked down in these cells. Due to the improved transfection efficiency of CCN1 siRNA\#1, this siRNA was used for the following experiments.
Effects of CCN1 knockdown on NO/eNOS and inflammation in PA-induced HUVECs. The levels of NO and eNOS were detected to evaluate endothelial function. As presented in Fig. 2A and B, PA decreased the levels of NO and p-eNOS compared with those in the control group, whereas CCN1 knockdown increased their levels. The results suggested that CCN1 knockdown could recover the inhibitory effects of PA on the levels of NO and eNOS in HUVECs. In order to assess whether CCN1 knockdown could alleviate the inflammation of PA-induced HUVECs, the expression levels of $\mathrm{p}$-IKK- $\beta$ and $\mathrm{p}-\mathrm{NF}-\kappa \mathrm{B}$ were determined in the present study. The results revealed that $\mathrm{p}-\mathrm{IKK}-\beta$ and $\mathrm{p}-\mathrm{NF}-\kappa \mathrm{B}$ were both elevated in the PA group compared with those in the control group, but were decreased in the PA + siRNA-CCN1\#1 group (Fig. 2C). Subsequently, the levels of inflammatory cytokines were measured using corresponding ELISA kits. The levels of TNF- $\alpha$, IL- $1 \beta$ and IL- 6 were elevated in PA-induced HUVECs compared with those in the control group, whereas CCN1 knockdown decreased the levels of these cytokines (Fig. 2D). These results indicated that CCN1 knockdown could alleviate inflammation of PA-induced HUVECs.

Effects of CCN1 knockdown on the apoptosis of PA-induced HUVECs. Apoptosis of HUVECs treated with PA was detected by TUNEL assay; the results revealed that PA promoted apoptosis of PA-induced HUVECs compared with that in the control group (Fig. 3A). In addition, the expression levels of apoptosis-associated proteins were detected by western blotting; the expression levels of Bax and cleaved caspase- 3 were enhanced after PA treatment, whereas the expression level of Bcl-2 were inhibited. Moreover, CCN1 knockdown reversed the aforementioned effects caused by PA compared with in the PA group (Fig. 3B). Overall, these 

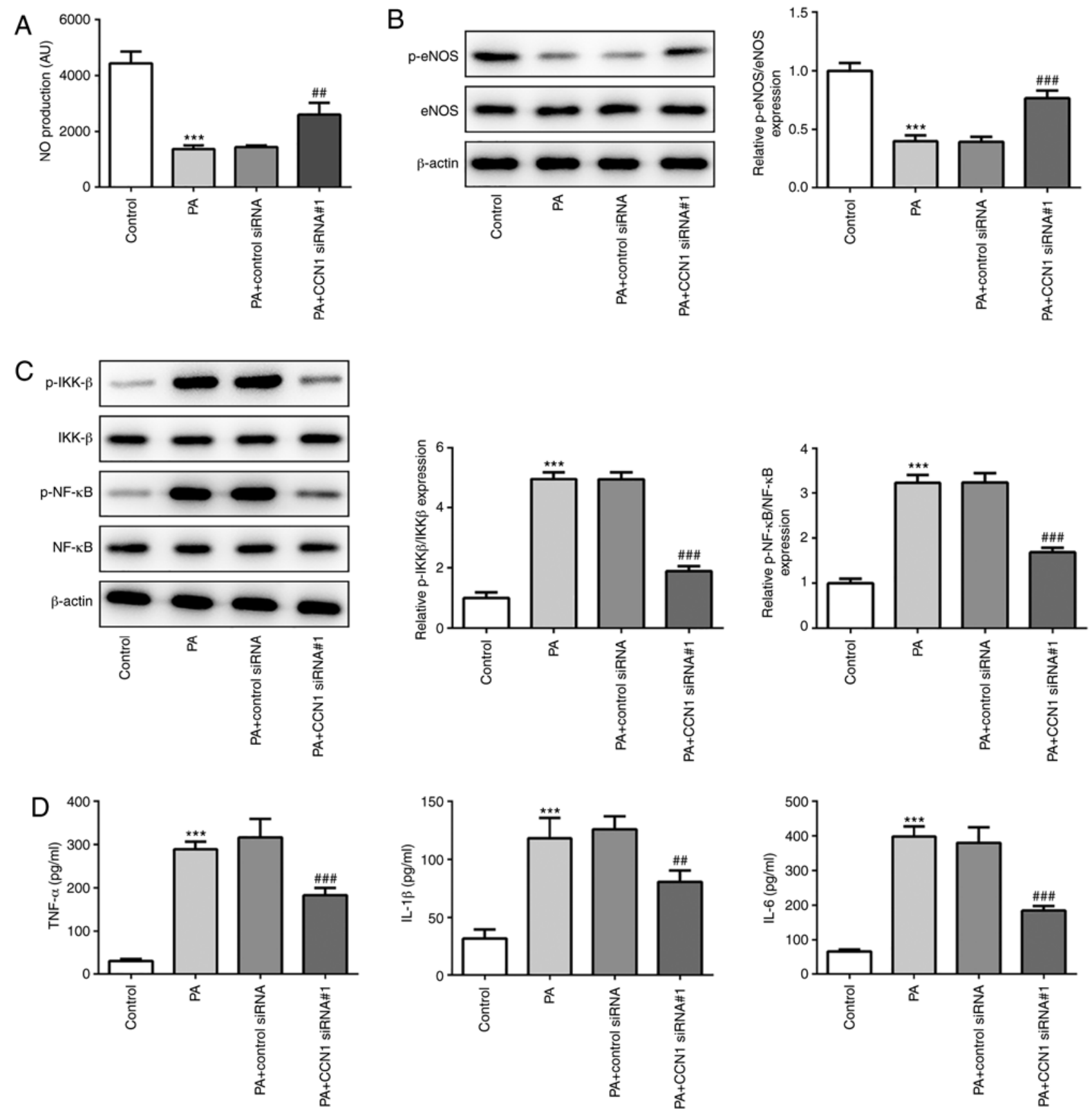

Figure 2. (A) NO production in each group. (B) Protein expression levels of $\mathrm{p}-\mathrm{eNOS}$ and eNOS in each group. (C) Protein expression levels of p-IKK- $\beta$, IKK- $\beta$,

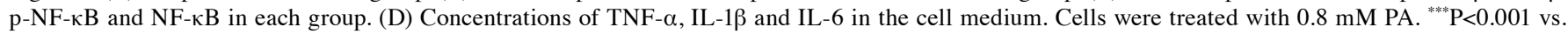
control group; ${ }^{\# \#} \mathrm{P}<0.001,{ }^{\# \#} \mathrm{P}<0.001$ vs. $\mathrm{PA}+$ control siRNA group. NO, nitric oxide; $\mathrm{p}$-, phosphorylated; eNOS, endothelial nitric oxide synthase; $\mathrm{PA}$, palmitic acid; CCN1, cysteine-rich angiogenic inducer 61; siRNA, small interfering RNA.

results suggested that CCN1 knockdown could inhibit cell apoptosis induced by PA.

Expression levels of DKK1 and $\beta$-catenin in PA-induced HUVECs. It has previously been reported that inhibition of the Wnt/ $\beta$-catenin signaling pathway can ameliorate endothelial cell injury (19); therefore, DKK1, which has been considered to obstruct the Wnt/ $\beta$-catenin signaling pathway (20), was investigated in the present study. The results revealed that the expression levels of $\beta$-catenin were gradually elevated, whereas those of DKK1 were decreased when HUVECs were exposed to increasing concentrations of PA compared with those in the control group (Fig. 4A), thus suggesting that PA may inhibit the expression levels of DKK1 and activate the Wnt/ $\beta$-catenin signaling pathway. Subsequently, OE-DKK1 and DKK1 siRNA were transfected into HUVECs, in order to regulate the expression of DKK1 (Fig. 4B-F); transfection of was successful, and siRNA-DKK1\#1 exhibited an improved knockdown effect.

Effects of DKK1 on the expression of CCN1 in PA-induced HUVECs. The effects of overexpression or silencing of DKK1 on the expression levels of CCN1 and $\beta$-catenin were subsequently assessed. The results of RT-qPCR and western 
A
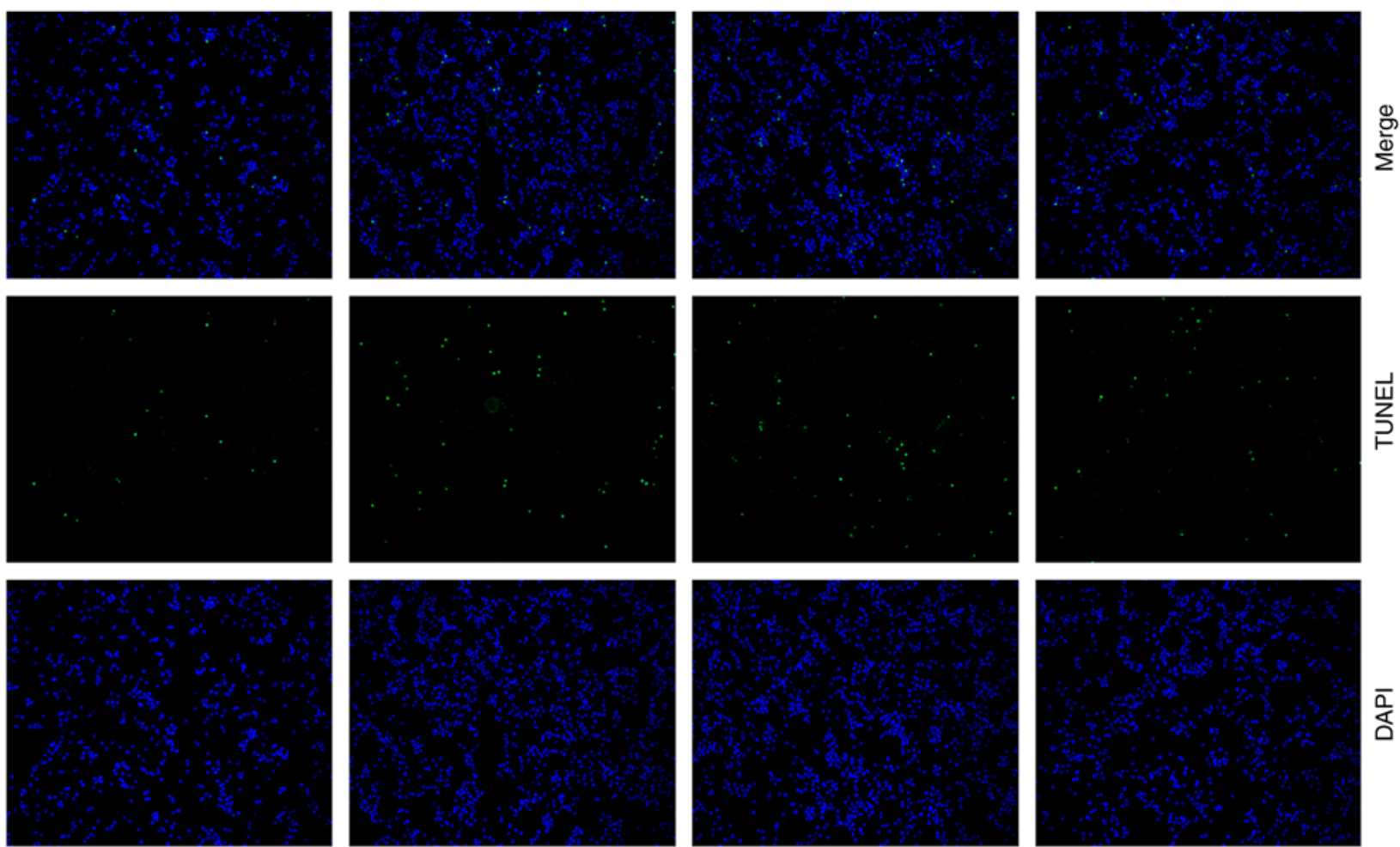

Control

PA

$\mathrm{PA}+$ control siRNA

$\mathrm{PA}+\mathrm{CCN} 1$ siRNA\#1

B
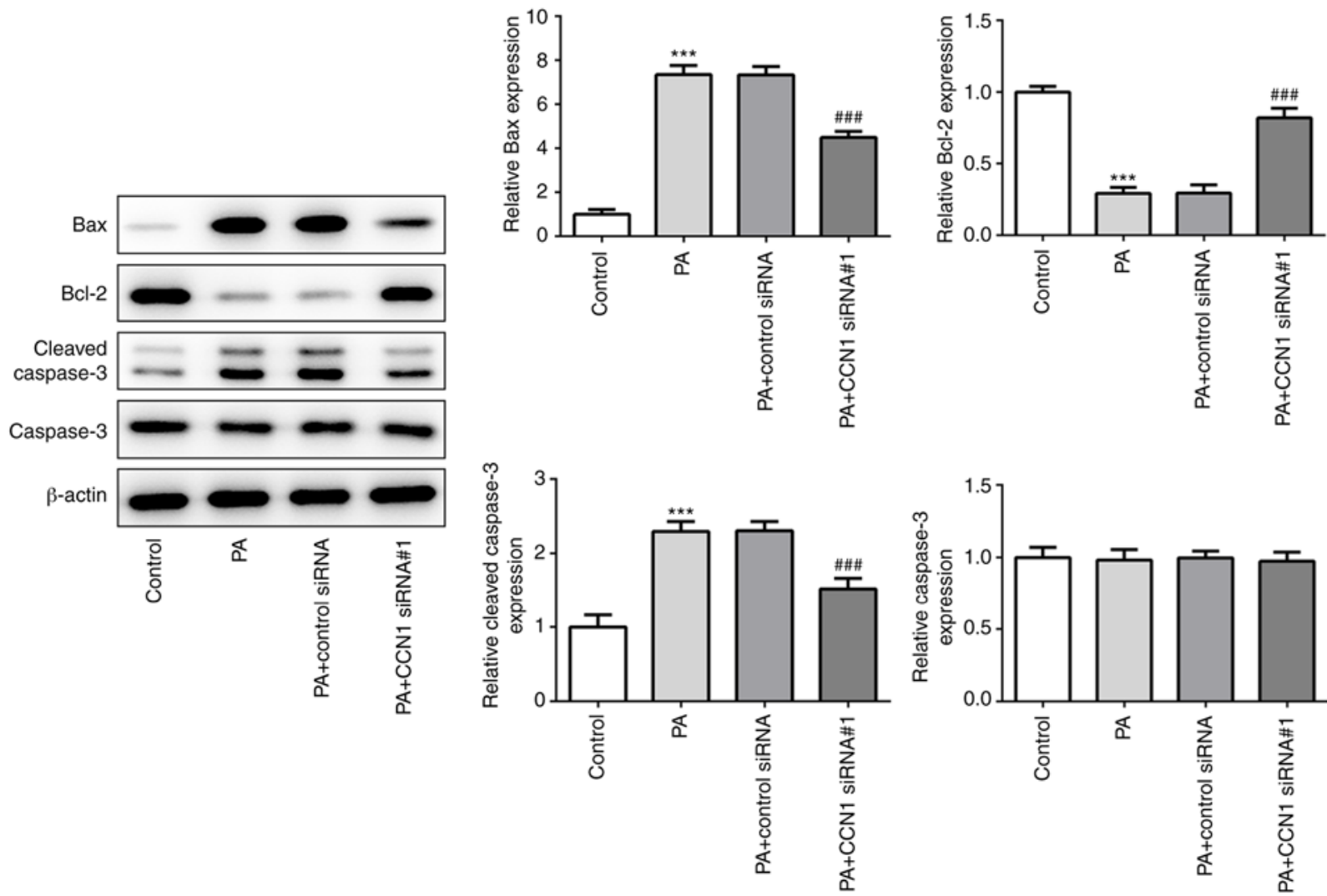

Figure 3. (A) TUNEL assay was performed to stain apoptotic cells (magnification, x100). (B) Protein expression levels of Bax, Bcl-2, cleaved-caspase-3 and caspase-3 in each group. Cells were treated with $0.8 \mathrm{mM}$ PA. ${ }^{* * *} \mathrm{P}<0.001$ vs. control group; ${ }^{\# \#} \mathrm{P}<0.001 \mathrm{vs}$. $\mathrm{PA}+$ control siRNA group. PA, palmitic acid; $\mathrm{CCN} 1$, cysteine-rich angiogenic inducer 61; siRNA, small interfering RNA.

blotting revealed that overexpression of DKK1 decreased the expression levels of $\mathrm{CCN} 1$ and $\beta$-catenin compared with those in the PA group; however, the opposite effects were detected when DKK1 was silenced (Fig. 5). These results suggested that activation and inhibition of the Wnt/ $\beta$-catenin signaling pathway may regulate the expression of CCN1. 
A

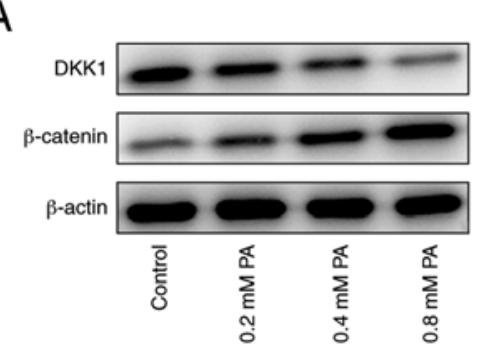

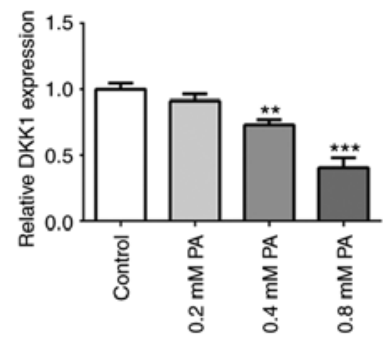

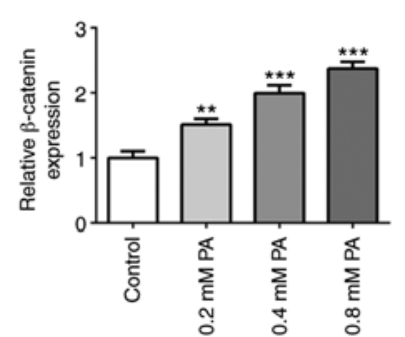

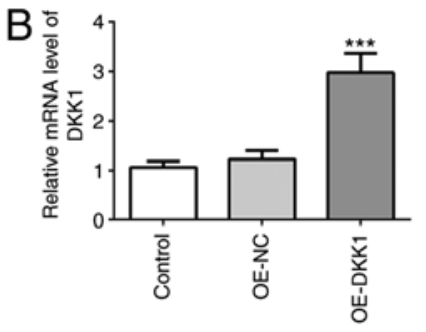
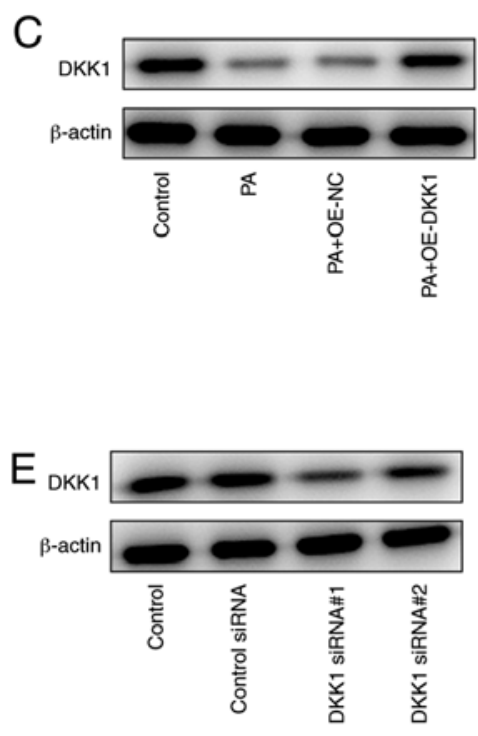
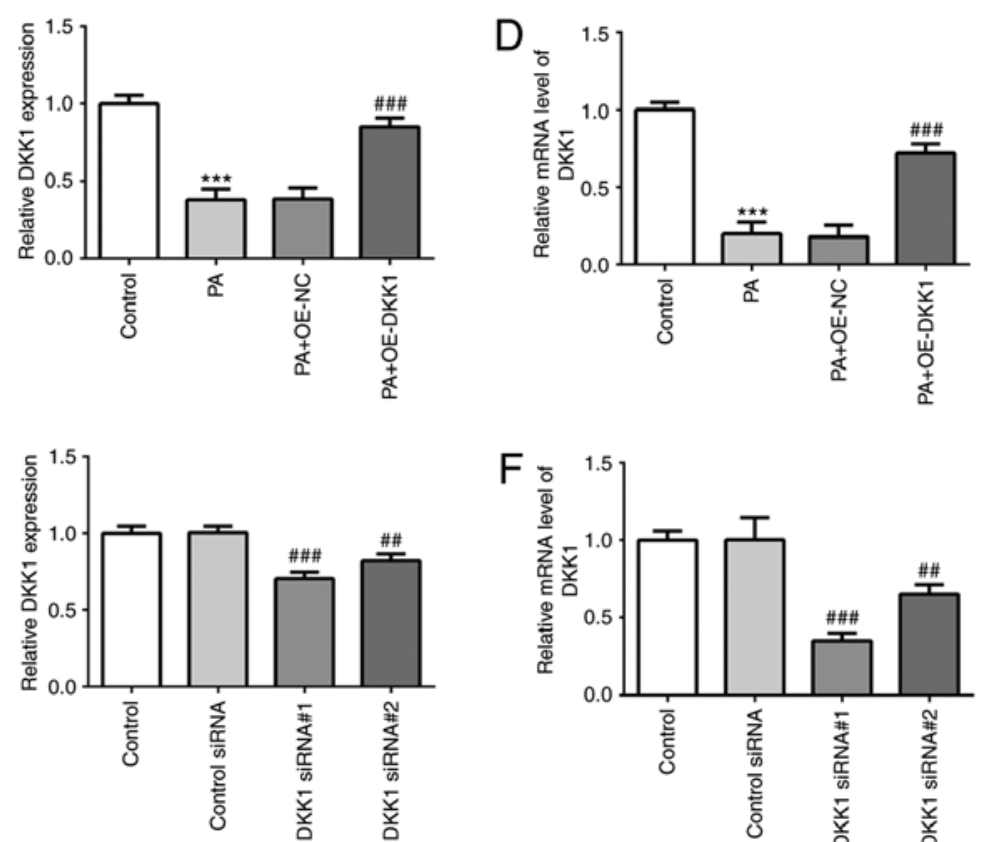

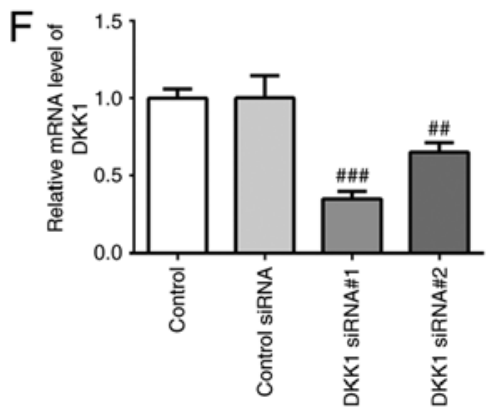

Figure 4. (A) Protein expression levels of DKK1 and $\beta$-catenin in HUVECs exposed to $0.2,0.4$ and $0.8 \mathrm{mM}$ PA for $24 \mathrm{~h} .{ }^{* *} \mathrm{P}<0.01,{ }^{* * * *} \mathrm{P}<0.001$ vs. control group (B) Transfection efficiency of OE-DKK1. ${ }^{* * *} \mathrm{P}<0.001$ vs. OE-NC group. (C) Protein and (D) mRNA expression levels of DKK1 in HUVECs exposed to $0.8 \mathrm{mM}$ PA with or without OE-DKK1. ${ }^{* * *} \mathrm{P}<0.001$ vs. control group; ${ }^{\# \#} \mathrm{P}<0.001$ vs. PA + OE-NC group. (E) Protein and (F) mRNA expression levels of DKK1 in HUVECs transfected with siRNA. ${ }^{\# \#} \mathrm{P}<0.001$, ${ }^{\# \#} \mathrm{P}<0.001$ vs. control siRNA group. DKK1, Dickkopf-1; PA, palmitic acid; HUVECs, human umbilical vein endothelial cells; OE, overexpression; NC, negative control; siRNA, small interfering RNA.

\section{Discussion}

CCN1 has been shown to be closely associated with atherosclerosis, based on its expression in diseased arteries, and has been reported to participate in cardiovascular development during embryogenesis (21-23). A previous study revealed that $\mathrm{CCN} 1$ was abnormally expressed in tissue injury and chronic diseases, suggesting its relevance in numerous pathologies (24). Notably, knockdown of CCN1 may have an important role in the alleviation of hyperlipemia, inflammation and the deterioration of atherosclerosis (7). In macrophages, inhibition of CCN1 expression via neutralizing antibodies or siRNAs decreased the lipid accumulation induced by ox-LDL (7). Furthermore, a previous study confirmed the role of CCN1 in the enhancement of endothelial cell apoptosis induced by TNF- $\alpha$ (2). These findings suggested that CCN1 may be a novel diagnostic marker and an effective target for the treatment of CVD. As endothelial dysfunction is a hallmark of the majority of cardiovascular risk factors and is associated with the initiation of atherosclerosis, PA was used to simulate the pathological conditions of endothelial dysfunction in the present study $(25,26)$. The results demonstrated that the expression levels of $\mathrm{CCN} 1$ were upregulated in PA-induced
HUVECs. Similarly, in a previous study, CCN1 was increased in mouse models under pathological conditions (27).

Endothelial dysfunction can also present as a decreased production or availability of $\mathrm{NO}$, which accounts for the risk of CVD and occurs prior to the development of atherosclerosis $(28,29)$. The results of the present study demonstrated that PA diminished the production of NO and the expression of p-eNOS, suggesting the occurrence of endothelial dysfunction in PA-induced HUVECs. After knockdown of CCN1 in PA-induced HUVECs, both NO and p-eNOS exhibited elevated levels, suggesting that the aberrant expression of CCN1 contributed to the occurrence of endothelial dysfunction. As inflammation is an important marker for endothelial dysfunction and CVD, the levels of inflammatory cytokines were evaluated in the present study (30). These cytokines exhibited elevated levels in PA-induced HUVECs. In agreement with previous studies that suggested CCN1 was a regulator of multiple cellular activities, such as migration, proliferation, inflammation and apoptosis $(23,31)$, the present study revealed that silencing CCN1 could alleviate inflammation and apoptosis. The results of the present study and of a previous study (32) provided an improved understanding on the previous evidence and suggested that 
A
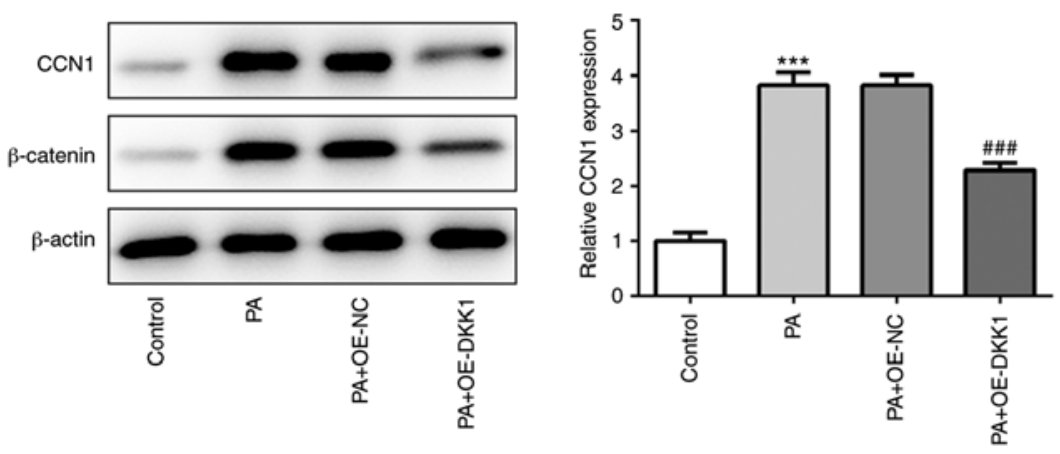

B

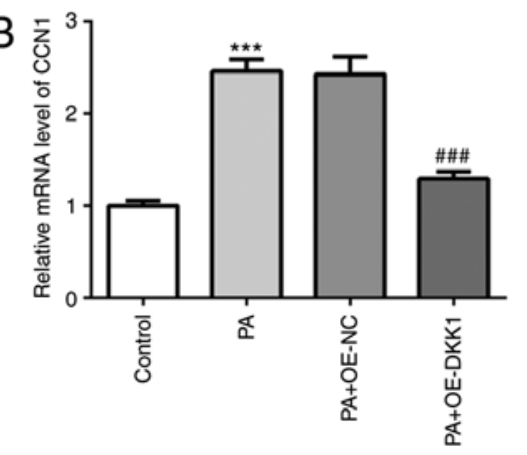

C

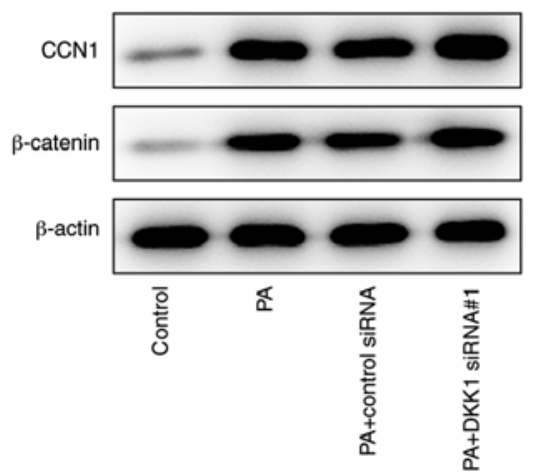

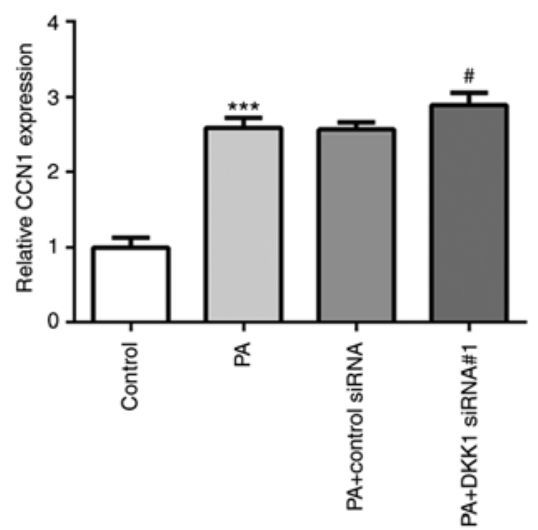

\section{D}

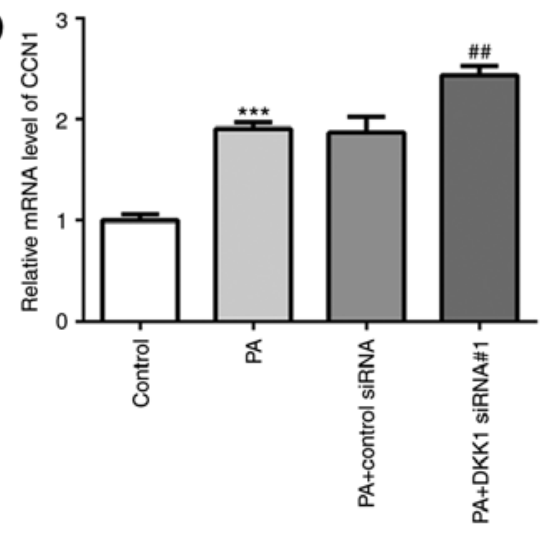

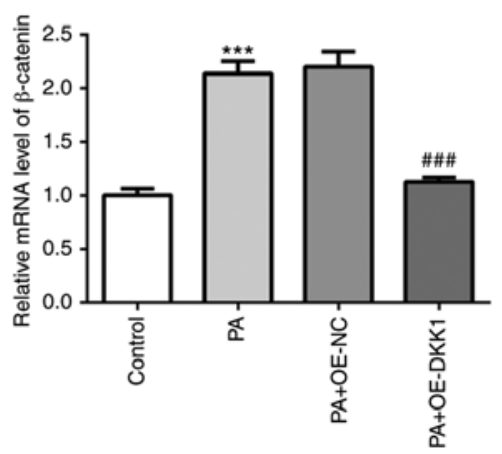
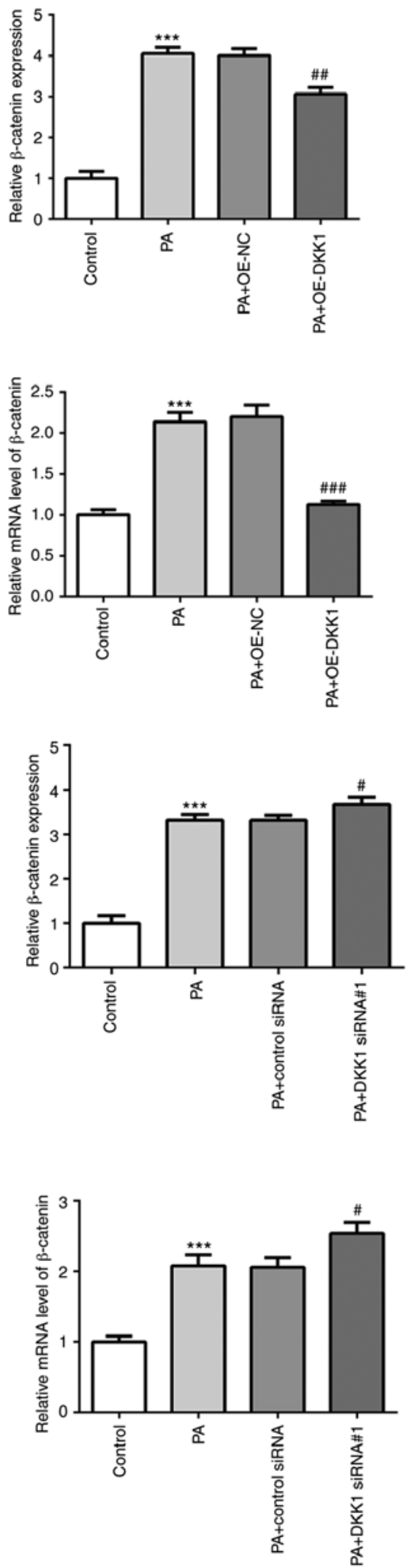

Figure 5. (A) Protein and (B) mRNA expression levels of CCN1 and $\beta$-catenin in HUVECs exposed to $0.8 \mathrm{mM}$ PA with or without OE-DKK1. ${ }^{* * *} \mathrm{P}<0.001$ vs. control group; ${ }^{\# \#} \mathrm{P}<0.001,{ }^{\# \# \#} \mathrm{P}<0.001$ vs. PA + OE-NC group. (C) Protein and (D) mRNA expression levels of CCN1 and $\beta$-catenin in HUVECs exposed to $0.8 \mathrm{mM}$ PA with or without siRNA. ${ }^{* * *} \mathrm{P}<0.001$ vs. control group; ${ }^{\#} \mathrm{P}<0.05,{ }^{\# \#} \mathrm{P}<0.01$ vs. $\mathrm{PA}+$ control siRNA group. CCN1, cysteine-rich angiogenic inducer 61 ; HUVECs, human umbilical vein endothelial cells; DKK1, Dickkopf-1; PA, palmitic acid; OE, overexpression; NC, negative control; siRNA, small interfering RNA.

profoundly altered CCN1 expression levels may affect the activities of inflammatory cytokines in vitro and in vivo.

The classical Wnt/ $\beta$-catenin signaling pathway has been implicated in various developmental processes, and mutations in this pathway have been observed in degenerative diseases, including Alzheimer's disease and in various types of cancer, such as non-small cell lung cancer (33-36). The Wnt/ $\beta$-catenin signaling pathway can be activated by highly conserved 
Wnt proteins (37). A recent study established the association between the $\mathrm{Wnt} / \beta$-catenin signaling pathway and atherosclerosis (38). In addition, research has revealed that activation of $\beta$-catenin could induce elevated expression levels of CCN1, and inhibition of Wnt/ $\beta$-catenin signaling could attenuate endothelial dysfunction $(19,39)$. Thus, the present study hypothesized that $\mathrm{Wnt} / \beta$-catenin signaling may regulate the expression of CCN1 to protect endothelial cells from PA-induced injury. DKK1, which can antagonize Wnt signaling by binding to LRP5/6 (34), was also assessed in the present study. In the present study, DKK1 expression was inhibited, whereas Wnt/ $\beta$-catenin signaling was activated when HUVECs were treated with increasing doses of PA. Overexpression of DKK1 inhibited activation of the Wnt/ $\beta$-catenin signaling in PA-treated HUVECs and further decreased the expression levels of CCN1. Conversely, silencing DKK1 activated the Wnt $/ \beta$-catenin signaling pathway and increased CCN1 expression. In conclusion, the present study provided evidence that DKK1/CCN1 may regulate PA-induced inflammation and apoptosis of HUVECs; however, the effects of DKK1/CCN1 need to be further verified in animal experiments, which may provide novel biomarkers for clinical diagnosis and therapeutic strategies for CVDs.

\section{Acknowledgements}

Not applicable.

\section{Funding}

This study was supported by the Lanzhou Talent Project for Innovation and Entrepreneurship (grant no. 2015-RC-12) and the Health Science and Technology Development Project of Lanzhou (grant no. 2019-002).

\section{Availability of data and materials}

The datasets used and/or analyzed during the current study are available from the corresponding author on reasonable request.

\section{Authors' contributions}

YRG and LW performed the experiments. YZW and ZKK analyzed the data. TXL and GWD drafted the manuscript and figures, and performed the experiments. YHD and DXX conceived and designed the study. All authors read and approved the final manuscript.

\section{Ethics approval and consent to participate}

Not applicable.

\section{Patient consent for publication}

Not applicable.

\section{Competing interests}

The authors declare that they have no competing interests.

\section{References}

1. Mozaffarian D: Trans fatty acids-effects on systemic inflammation and endothelial function. Atheroscler Suppl 7: 29-32, 2006.

2. Zhang J, Wu G and Dai $\mathrm{H}$ : The matricellular protein $\mathrm{CCN} 1$ regulates TNF- $\alpha$ induced vascular endothelial cell apoptosis. Cell Biol Int 40: 1-6, 2016.

3. Nilsson J: Atherosclerosis-the molecular background. Lakartidningen 88: 127-129, 1991 (In Swedish).

4. Tada H, Takamura M and Kawashiri MA: What is the mechanism of genetic contributions to the development of atherosclerosis? Atherosclerosis 307: 72-74, 2020.

5. Emre Y and Imhof BA: Matricellular protein CCN1/CYR61: A new player in inflammation and leukocyte trafficking. Semin Immunopathol 36: 253-259, 2014.

6. Wong M, Kireeva ML, Kolesnikova TV and Lau LF: Cyr61, product of a growth factor-inducible immediate-early gene, regulates chondrogenesis in mouse limb bud mesenchymal cells. Dev Biol 192: 492-508, 1997.

7. Zhao JF, Chen HY, Wei J, Jim Leu SJ and Lee TS: CCN family member 1 deregulates cholesterol metabolism and aggravates atherosclerosis. Acta Physiol (Oxf) 225: e13209, 2019.

8. Mielenz M: Invited review: Nutrient-sensing receptors for free fatty acids and hydroxycarboxylic acids in farm animals. Animal 11: 1008-1016, 2017.

9. Briggs MA, Petersen KS and Kris-Etherton PM: Saturated fatty acids and cardiovascular disease: Replacements for saturated fat to reduce cardiovascular risk. Healthcare (Basel) 5: 29, 2017.

10. Yang L, Guan G, Lei L, Lv Q, Liu S, Zhan X, Jiang Z and Gu X: Palmitic acid induces human osteoblast-like Saos-2 cell apoptosis via endoplasmic reticulum stress and autophagy. Cell Stress Chaperones 23: 1283-1294, 2018.

11. Miyamoto J, Hasegawa S, Kasubuchi M, Ichimura A, Nakajima A and Kimura I: Nutritional signaling via free fatty acid receptors. Int J Mol Sci 17: 450, 2016.

12. Jiang $H$, Liang $C$, Liu $X$, Jiang Q, He Z, Wu J, Pan X, Ren $Y$, Fan M, Li M and Wu Z: Palmitic acid promotes endothelial progenitor cells apoptosis via $\mathrm{p} 38$ and JNK mitogen-activated protein kinase pathways. Atherosclerosis 210: 71-77, 2010.

13. Leroy C, Tricot S, Lacour B and Grynberg A: Protective effect of eicosapentaenoic acid on palmitate-induced apoptosis in neonatal cardiomyocytes. Biochim Biophys Acta 1781: 685-693, 2008.

14. Chen X, Liu L, Palacios G, Gao J, Zhang N, Li G, Lu J, Song T, Zhang Y and Lv H: Plasma metabolomics reveals biomarkers of the atherosclerosis. J Sep Sci 33: 2776-2783, 2010.

15. Geng J, Xu H, Yu X, Xu G, Cao H, Lin G and Sui D: Rosuvastatin protects against oxidized low-density lipoprotein-induced endothelial cell injury of atherosclerosis in vitro. Mol Med Rep 19: 432-440, 2019.

16. Chen P, Liu H, Xiang H, Zhou J, Zeng Z, Chen R, Zhao S, Xiao J, Shu Z, Chen S and Lu H: Palmitic acid-induced autophagy increases reactive oxygen species via the $\mathrm{Ca}^{2+} / \mathrm{PKC} \alpha / \mathrm{NOX} 4$ pathway and impairs endothelial function in human umbilical vein endothelial cells. Exp Ther Med 17: 2425-2432, 2019.

17. Tajadura V, Hansen MH, Smith J, Charles H, Rickman M, Farrell-Dillon K, Claro V, Warboys C and Ferro A: $\beta$-catenin promotes endothelial survival by regulating eNOS activity and flow-dependent anti-apoptotic gene expression. Cell Death Dis 11: 493, 2020.

18. Livak KJ and Schmittgen TD: Analysis of relative gene expression data using real-time quantitative PCR and the 2(-Delta Delta C(T)) method. Methods 25: 402-408, 2001.

19. Ma S, Yao S, Tian H, Jiao P, Yang N, Zhu P and Qin S: Pigment epithelium-derived factor alleviates endothelial injury by inhibiting Wnt/beta-catenin pathway. Lipids Health Dis 16: 31, 2017.

20. Ali H, Zmuda JM, Cvejkus RK, Kershaw EE, Kuipers AL, Oczypok EA, Wheeler V, Bunker $\mathrm{CH}$ and Miljkovic I: Wnt pathway inhibitor DKK1: A potential novel biomarker for adiposity. J Endocr Soc 3: 488-495, 2019.

21. Mo FE and Lau LF: The matricellular protein CCN1 is essential for cardiac development. Circ Res 99: 961-969, 2006.

22. Hsu PL, Chen JS, Wang CY, Wu HL and Mo FE: Shear-induced CCN1 promotes atheroprone endothelial phenotypes and atherosclerosis. Circulation 139: 2877-2891, 2019.

23. Lau LF: CCN1/CYR61: The very model of a modern matricellular protein. Cell Mol Life Sci 68: 3149-3163, 2011.

24. Jun JI and Lau LF: Taking aim at the extracellular matrix: CCN proteins as emerging therapeutic targets. Nat Rev Drug Discov 10: 945-963, 2011. 
25. Otsuka F, Finn AV, Yazdani SK, Nakano M, Kolodgie FD and Virmani R: The importance of the endothelium in atherothrombosis and coronary stenting. Nat Rev Cardiol 9: 439-453, 2012.

26. Daiber A, Xia N, Steven S, Oelze M, Hanf A, Kröller-Schön S, Münzel T and Li H: New therapeutic implications of endothelial nitric oxide synthase (eNOS) Function/Dysfunction in cardiovascular disease. Int J Mol Sci 20: 187, 2019.

27. Ju L, Sun Y, Xue H, Chen L, Gu C, Shao J, Lu R, Luo X, Wei J, Ma X and Bian Z: CCN1 promotes hepatic steatosis and inflammation in non-alcoholic steatohepatitis. Sci Rep 10: 3201, 2020.

28. Ghosh A, Gao L, Thakur A, Siu PM and Lai CWK: Role of free fatty acids in endothelial dysfunction. J Biomed Sci 24: 50, 2017.

29. Hadi HA, Carr CS and Al Suwaidi J: Endothelial dysfunction: Cardiovascular risk factors, therapy, and outcome. Vasc Health Risk Manag 1: 183-198, 2005.

30. Kaptoge S, Seshasai SR, Gao P, Freitag DF, Butterworth AS, Borglykke A, Di Angelantonio E, Gudnason V, Rumley A, Lowe GD, et al: Inflammatory cytokines and risk of coronary heart disease: New prospective study and updated meta-analysis. Eur Heart J 35: 578-589, 2014.

31. Chiodoni C, Colombo MP and Sangaletti S: Matricellular proteins: From homeostasis to inflammation, cancer, and metastasis. Cancer Metastasis Rev 29: 295-307, 2010.

32. Chen CC, Juric V and Lau LF: The extracellular matrix protein CCN1 dictates TNFalpha and FasL cytotoxicity in vivo. Adv Exp Med Biol 691: 595-603, 2011.
33. de Jaime-Soguero A, Abreu de Oliveira WA and Lluis F: The pleiotropic effects of the canonical Wnt pathway in early development and pluripotency. Genes (Basel) 9: 93, 2018.

34. Nusse $\mathrm{R}$ and Clevers $\mathrm{H}$ : Wnt $/ \beta$-catenin signaling, disease, and emerging therapeutic modalities. Cell 169: 985-999, 2017.

35. Lin S, Zhen Y, Guan Y and Yi H: Roles of Wnt/ $\beta$-catenin signaling pathway regulatory long non-coding RNAs in the pathogenesis of non-small cell lung cancer. Cancer Manag Res 12: 4181-4191, 2020.

36. Jia L, Piña-Crespo J and Li Y: Restoring Wnt/ $\beta$-catenin signaling is a promising therapeutic strategy for Alzheimer's disease. Mol Brain 12: 104, 2019.

37. Herr P and Basler K: Porcupine-mediated lipidation is required for Wnt recognition by Wls. Dev Biol 361: 392-402, 2012.

38. Foulquier S, Daskalopoulos EP, Lluri G, Hermans KCM, Deb A and Blankesteijn WM: WNT signaling in cardiac and vascular disease. Pharmacol Rev 70: 68-141, 2018.

39. Li ZQ, Ding W, Sun SJ, Li J, Pan J, Zhao C, Wu WR and Si WK: Cyr61/CCN1 is regulated by Wnt/ $\beta$-catenin signaling and plays an important role in the progression of hepatocellular carcinoma. PLoS One 7: e35754, 2012.

This work is licensed under a Creative Commons Attribution-NonCommercial-NoDerivatives 4.0 International (CC BY-NC-ND 4.0) License. 